\title{
La naturaleza de la ciencia y la tecnología. Una experiencia para desarrollar el pensamiento crítico
}

\author{
The nature of science and technology. An experience to develop Critical Thinking
}

A natureza da ciência e tecnologia. Uma experiência para desenvolver o Pensamento

\author{
Crítico
}

Vanessa Ortega-Quevedo

Cristina Gil Puente ${ }^{2}$

Recibido: enero de 2019

Aceptado: abril de 2019

Para citar este artículo: Ortega-Quevedo, V.; Gil-Puente, C. (2019). La naturaleza de la ciencia y la tecnología. Una experiencia para desarrollar el pensamiento crítico. Revista Científica, 35(2), 167-182. Doi: https://doi. org/10.14483/23448350.14095

\section{Resumen}

La investigación que se presenta tiene como objetivo principal el desarrollo del pensamiento crítiCo (PC) de estudiantes de educación primaria y la enseñanza de temáticas relacionadas con la naturaleza de la ciencia y la tecnología (NdCyT). Se desarrolla y pone en funcionamiento una secuencia de enseñanza-aprendizaje realizada ad hoc; tanto el análisis como la evaluación de los resultados obtenidos se efectúan a través de un enfoque cuantitativo. La muestra del estudio se conformó por 130 participantes de $6^{\circ}$ curso de educación primaria. Los instrumentos de evaluación utilizados son dos adaptaciones: la primera del cuestionario de opinión sobre ciencia, tecnología y sociedad; y la segunda de una traducción de la prueba de Halpern. Como resultado, se observa que los participantes en el estudio manifiestan mejoras significativas en las habilidades de PC y las concepciones relativas a la $\mathrm{NdCyT}$ estudiadas. En consecuencia, la experiencia se considera exitosa.

Palabras clave: argumentación, aspectos no epistémicos, energía, educación básica, probabilidad, razonamiento verbal.

\begin{abstract}
The main objective of the research presented is the development of Critical Thinking (CT) of Primary Education students and the teaching of topics related to the Nature of Science and Technology (NoS\&T). A Teaching-Learning Sequence is developed and implemented, both the analysis and the evaluation of the results obtained is done through a quantitative approach. The study is carried out with a sample of 130 participants of the 6th grade of Elementary Education. The evaluation instruments that
\end{abstract}


used are two adaptations, the first is the Opinion Questionnaire on Science, Technology and Society, and the second is a Spanish version of the Halpern test. As a result, we observed that the participants in the study show significant improvements in PC skills and conceptions related to the NoS\&T studied. Consequently, the experience is considered successful. Keywords: Elementary education, science education, no epistemic aspects, argumentation, verbal reasoning, probability, energy.

\section{Resumo}

O principal objetivo da pesquisa apresentada é o desenvolvimento do Pensamento Crítico (PC) de alunos do Ensino Fundamental e do ensino de tópicos relacionados à Natureza da Ciência e Tecnologia (NdCyT). Uma seqüência ad hoc de ensino-aprendizagem é desenvolvida e implementada, tanto a análise quanto a avaliação dos resultados obtidos são realizadas por meio de uma abordagem quantitativa. A amostra do estudo é composta por 177 participantes do $6 .^{\circ}$ ano do Ensino Fundamental. Os instrumentos de avaliação utilizados são duas adaptações: a primeira do Questionário de Opinião sobre Ciência, Tecnologia e Sociedade, e a segunda de uma tradução do teste de Halpern. Como resultado, nós observamos que os participantes do estudo apresentam melhorias significativas nas habilidades e concepções de PC relacionadas ao NdCyT estudado. Consequentemente, a experiência é considerada bem sucedida.

Palavras-chaves: educação básica, educação científica, aspectos não-epistêmicos, argumentação, raciocínio verbal, probabilidade, energia.

\section{Introducción}

La sociedad actual demanda que la educación promueva el desarrollo de capacidades como las de pensamiento crítico (PC). El informe Work Skills 2020 elaborado por el instituto de investigación de la Universidad de Phoenix (Davies, Fidler y Gorbis, 2011) prevé que en 2020 la demanda de capacidades cognitivas y sociales de carácter superior, como el PC, serán solicitadas para ocupar la mayor parte de los puestos de trabajo. Es por ello necesario que el sistema educativo se haga cargo de formar al alumnado para que desarrollen sus habilidades de PC: aprender a pensar (realizar procesos de identificación, análisis, evaluación, etc. de la información) y a obtener una autonomía intelectual mediante el desarrollo de los procedimientos propios del componente disposicional del PC. Es más, el desarrollo de esta competencia no revierte positivamente solo en la vida adulta de los jóvenes, sino que influye de la misma forma en su rendimiento académico. Estos argumentos ilustran la relevancia de estudiar el desarrollo de competencias de PC en la actualidad (da Silva y Rodríguez, 2011; López, 2012; Franco, Almeida y Morales, 2014).

En esta misma línea cabe destacar el Programa Marco de Investigación e Innovación de la Unión Europea Horizonte 2020 (s. f.) cuyo objetivo es "promover y facilitar la comprensión de la Investigación e Innovación responsable mediante acciones como el desarrollo de los intereses y capacidades de los ciudadanos hacia la ciencia, de forma que puedan participar activamente en actividades científicas". Estos planteamientos se justifican en el contexto social actual, donde en el mundo globalizado en el que vivimos los impactos científicos son constantes y la presencia de la ciencia repercute en factores sociales trascendentales como la política, la educación, la cultura, el ocio, etc. (Vázquez y Manassero, 2012). De ahí que surja la necesidad de plantear una nueva forma de entender la enseñanza de las ciencias que esté en consonancia con la nueva realidad social y que permita entender esta de una forma inclusiva (Solbes, Montserrat y Furió, 2007; Vázquez y Manassero, 2012, Martín, Prieto y Jiménez, 2015).

A partir de las reflexiones expuestas, se justifica la necesidad de introducir el desarrollo de las competencias científica, tecnológica y de pensamiento crítico en la educación obligatoria. En concreto esta investigación se centra en su desarrollo en la etapa de educación primaria, en la que el marco legislativo español respalda esta decisión, ya que: la Ley Orgánica 8/2013 de 9 de diciembre para la 
mejora de la calidad educativa (Lomce), establece que: "las habilidades cognitivas, siendo imprescindibles, no son suficientes; es necesario adquirir desde edades tempranas competencias transversales, como el Pensamiento Crítico" (p. 97860).

El Real Decreto 126/2014 del 28 de febrero, por el que se establece el currículo básico de la educación primaria, implanta siete competencias clave para el desarrollo personal entre las cuales se encuentra la competencia matemática y competencias básicas en ciencia y tecnología. En otras palabras, la legislación determina que durante la enseñanza obligatoria es necesario que los estudiantes desarrollen competencias básicas en ciencia y tecnología que les permita llegar a una alfabetización científica ciudadana.

\section{Marco teórico}

A pesar de que el PC se ha estudiado desde hace años, el amplio interés global es relativamente reciente. Este resurgimiento puede atribuirse a los cambios que ha experimentado la sociedad, y a los cambios que demanda la enseñanza del siglo XXI, como más y mejor instrucción en habilidades de pensar bien (Norris, 1985). En esta línea, las capacidades cognitivas de alto nivel incluidas dentro del PC (análisis, razonamiento, capacidad de resolución de problemas, argumentación, formulación de hipótesis, etc.) se identifican con las destrezas propias del pensamiento científico (Manassero y Vázquez, 2017). A Juicio de Gold (2002), la razón por la que los estudiantes no son capaces de apreciar el valor de las pruebas científicas reside en su incapacidad de poner en práctica las capacidades de pensamiento científico o PC. Esta coincidencia hace que la educación científica sea un contexto apropiado para educar el PC de los estudiantes y viceversa.

En este sentido, la presente investigación contextualiza el desarrollo del PC dentro del ámbito de la educación científica reconocido como $\mathrm{Nd}$ CyT, pues las cuestiones relativas a dicho constructo conforman uno de los elementos clave de la alfabetización científico-tecnológica (CT)
(Acevedo-Díaz, Aragón-Méndez, García-Carmona, 2018; Acevedo-Díaz y García-Carmona 2016; Vázquez-Alonso y Manassero-Mas, 2018). Ahora bien, antes de continuar es preciso determinar qué se entiende por PC, alfabetización CT y NdCyT.

Existen múltiples definiciones de PC, algunas de las más representativas del concepto (Facione, 1990; Ennis, 1996; Paul, 2005; Paul y Elder, 2002) lejos de ser excluyentes se complementan dando lugar a una definición completa que contempla el PC desde distintas perspectivas. De este modo, el PC se conforma a partir de un conjunto de procesos que se emplean deliberadamente a fin de concluir cuestiones acerca de distintas temáticas. Al aplicar estos procesos se descomponen, sintetizan y evalúan de forma reflexiva fragmentos de información o problemas, para determinar una conclusión o solución sobre estos. Asimismo, es necesario aplicar una reflexión sobre los propios procesos de razonamiento, con el fin de evaluar los mismos y concluir si son correctos o mejorables (Valenzuela y Nieto 2008, da Silva y Rodríguez, 2011).

Se entiende por alfabetización CT la capacidad de una persona para comprender artículos sobre ciencia y tecnología y poder participar en debates sociales acerca de estos; posicionarse con conocimiento y ética ante una decisión política que afecte a un aspecto CT; o para valorar la calidad de la información CT, entre otras cuestiones (Pedrinaci, Caamaño, Cañal y de Pro, 2012; Vázquez y Manassero; 2012). Una vez delimitado este concepto, se exponen los dos componentes que forman esta alfabetización: 1) "la comprensión 'de' la ciencia (los tradicionales conocimientos sobre hechos, conceptos, principios y procesos de la ciencia)" (Manassero y Vázquez, 2017, p. 509);, y 2) "la comprensión 'acerca' de la ciencia [...] formado por contenidos interdisciplinares de historia, filosofía y sociología de la ciencia y tecnología, pero también de psicología, economía, política, ética, etc." (Manassero y Vázquez, 2017, p. 510). Este último componente está íntimamente relacionado con el concepto $\mathrm{Nd}$ CyT que se aborda en esta investigación. 
Para esclarecer que se entiende por NdCyT en esta investigación hay que partir del concepto de naturaleza de la ciencia $(\mathrm{NdC})$. La ciencia es dinámica, por lo tanto, definir con precisión el concepto de $\mathrm{NdC}$ es complejo. En los últimos decenios, los acuerdos sobre $\mathrm{NdC}$ se han centrado en contenidos epistemológicos (Abd-El-Khalick, 2012), sin prestar demasiada atención a aspectos sociológicos, culturales, etc.

No obstante, en esta investigación se entiende que la $\mathrm{NdC}$ "es un meta-conocimiento sobre la ciencia, que surge de las reflexiones interdisciplinares hechas por expertos en filosofía, historia y sociología de la ciencia, así como por algunos científicos y educadores de ciencias" (Acevedo-Díaz, García-Carmona y Aragón, 2016, p. 914). Esta concepción de la $\mathrm{NdC}$ incluye aspectos epistémicos, aspectos de sociología interna y externa de la ciencia, aspectos que incluyen la relación entre ciencia, tecnología y sociedad (Acevedo-Díaz, García-Carmona y Aragón, 2018). Así pues, a esta visión holística de la $\mathrm{NdC}$ se le incluye la tecnología y una dimensión de la ciencia como elemento capaz de dar solución a problemas existentes en la sociedad actual (instrumental) dentro del concepto $\mathrm{NdC}$, el cual se pasa a denominar NdCyT como forma de resaltar su analogía con el concepto tecnociencia, así como las relaciones ciencia-tecnología-sociedad (Vázquez, Manassero y de Talavera, 2010; Manassero y Vázquez, 2017).

Como último punto de este marco teórico, es preciso resaltar los enfoques de enseñanza de los que esta investigación parte para fomentar el desarrollo del PC y de la alfabetización científica.

El desarrollo del PC se torna complejo, de modo que para efectuar una buena enseñanza de este se deben tener en cuenta de forma específica cuatro aspectos fundamentales: las estrategias, el metaconocimiento, las disposiciones y la práctica (Saiz, 2002). Por lo que es preciso poner en marcha estrategias que permitan guiar el aprendizaje e identificar ciertas habilidades de PC; dejar espacios para la reflexión sobre los procesos de pensamiento (es más importante el proceso interno de pensamiento que el producto); y motivar al alumnado para que este predispuesto a poner en práctica habilidades de PC concretas y practicar el uso de las mismas.

Finalmente, el desarrollo de la alfabetización científica se procura mediante la enseñanza de temas de NdCyT desde un planteamiento explícito, pues mejora la comprensión sobre NdCyT más que los métodos implícitos (Deng, Chen, Tsai y Chai, 2011); y reflexivo debido a la realización de actividades meta-cognitivas de autorreflexión (conexión con habilidades de PC) por los estudiantes.

El objeto de estudio de la presente investigación es el desarrollo del PC y la mejora de las concepciones de los discentes sobre NdCyT a través de la implementación de una secuencia de enseñanza-aprendizaje (SEA) en educación primaria. Tanto el PC como la NdCyT son constructos muy amplios, su trabajo se ha concretado en cuatro temas de $\mathrm{Nd}$ CyT relacionados con la influencia de la ciencia y la tecnología en la sociedad y con la construcción social de la tecnología (quién debería decidir sobre los asuntos científicos, existencia de equilibrios entre efectos positivos y negativos de la ciencia y la tecnología, decisiones sobre la implementación de una nueva tecnología, control del desarrollo tecnológico por la ciudadanía); y tres habilidades de pensamiento crítico (razonamiento verbal, argumentación y probabilidad). Diseñar, de acuerdo con el cuerpo teórico, una SEA que permita a estudiantes de 11 y 12 años desarrollar determinadas habilidades de PC, mientras aprenden cuestiones relacionadas con la $\mathrm{NdCyT}$ que les permitan mejorar sus concepciones acerca de esta. Realizar una evaluación de la mejora de las habilidades de PC y las concepciones de $\mathrm{NdCyT}$ que manifiesta poseer el alumnado, con el fin de comprobar la evolución de los discentes tras la puesta en práctica de la SEA, así como la validez de la misma.

\section{Metodología}

El diseño de la investigación es cuasi-experimental con una estructura de tipo panel 
(pre-test-intervención-post-test). La metodología aplicada es cuantitativa, así como los instrumentos de evaluación.

La realización de la investigación consta de tres fases llevadas a cabo durante tres meses: cumplimentación de dos cuestionarios a modo de diagnóstico inicial (pre-test) por parte del alumnado, implementación de una SEA por parte del investigador (tres sesiones de una hora de duración que pueden flexibilizarse según las necesidades del grupo) y cumplimentación de los cuestionarios a modo de evaluación final (post-test).

\section{Contexto}

La experiencia desarrollada se ha llevado a cabo en el área de Ciencias de la Naturaleza como un taller de introducción y repaso de contenidos en varios grupos de $6^{\circ}$ de educación primaria pertenecientes a distintos centros educativos públicos de Segovia. Dichos centros acogen a una población muy dispar, de modo que el alumnado posee perfiles académicos y socioculturales muy diferentes. Es importante resaltar que el docente que aplica las sesiones de la SEA en todos los centros es el mismo, reduciendo de esta forma las variables extrañas relacionadas con la aplicación didáctica.

\section{Muestra}

Los participantes de este estudio fueron 130 estudiantes de $6^{\circ}$ de educación primaria pertenecientes a cinco centros educativos públicos de Segovia (tabla 1).

\section{Descripción de la propuesta didáctica}

La SEA se puede implantar por sus contenidos y el desarrollo psicoevolutivo del alumnado en los cursos $5 .^{\circ}$ y $6 .^{\circ}$ de educación primaria, en el área de Ciencias de la Naturaleza. Esta establece los contenidos correspondientes a los factores de estudio mediante el trabajo de contenidos previstos en el currículo de educación primaria (Real Decreto 126, 2014). En concreto, la programación aborda distintos contenidos: 1) contenidos curriculares, la energía; 2) cuatro temáticas de NdCyT relacionadas con aspectos de sociología interna y externa de la ciencia seleccionados por su afinidad con los contenidos curriculares, lo cual promueve la contextualización de estos y una visión no distorsionada de la ciencia y la tecnología; 3 ) las capacidades de PC señaladas anteriormente. El tratamiento de todos estos contenidos se trabaja de una forma explícita, pues son contenidos planificados y evaluables dentro de la SEA.

\section{Metodología didáctica}

El planteamiento metodológico empleado no se corresponde con un modelo didáctico, sino que emplea estrategias propias de distintos modelos para diseñar un proceso de enseñanza-aprendizaje óptimo de las ciencias (Garrido, Perales y Galdón, 2007):

- Implementar actividades con cierto carácter lúdico que permitan reconocer el nivel de conocimientos previos del alumnado (sobre los

Tabla 1. Descripción de la muestra.

\begin{tabular}{clclc}
\hline $\begin{array}{c}\text { Centros } \\
\text { educativos }\end{array}$ & $\begin{array}{l}\text { Nivel sociocultural de las } \\
\text { familias }\end{array}$ & $\begin{array}{c}\text { Líneas del } \\
\text { centro }\end{array}$ & Líneas participantes & Total de participantes \\
1 & Medio & 2 & 2 Grupos experimentales & 43 \\
2 & Bajo & 2 & 2 Grupos experimentales & 36 \\
3 & Medio-bajo & 2 & 1 Grupo experimental & 19 \\
4 & Medio-alto & 1 & 1 Grupo experimental & 19 \\
5 & Medio & 2 & 1 Grupo control & 13 \\
\end{tabular}

Fuente: Elaboración propia de los autores. 
contenidos curriculares), así como que estos extraigan y exploren dichos conocimientos y se enganchen a la actividad (evaluación inicial complementaría a la realizada en la fase pre-test).

- Realizar una transmisión de los contenidos (explicar) para construir una buena base teórica antes de iniciar procesos y capacidades cognitivas complejas.

- Proporcionar a los discentes elementos que les ayuden a descubrir por sí mismos la trascendencia de los contenidos teóricos (explorar).

- Poner en práctica estrategias de comunicativas de la enseñanza, ya que se entiende que a través del diálogo los discentes indagan y transforman el conocimiento (elaborar y extender) (Wells, 2001).

- Determinar el papel del docente como presentador de contenidos y guía en la construcción del aprendizaje. Este rol es determinante, pues es la clave para mediar en los diálogos y reconducir al alumnado para que pueda identificar sus problemas o errores y construir un conocimiento lo más próximo posible a las concepciones correctas sobre la ciencia (González, 2015).

- Evaluar, de acuerdo con los planteamientos didácticos, contenidos innovadores en la SEA, pues de lo contrario dichos planteamientos quedarían invalidados (Dochy, Segers y Dierick, 2002). Con el fin de atender a esta premisa, se establece una forma de evaluación formativa inherente a todo el proceso de enseñanza-aprendizaje (de los contenidos curriculares, de las concepciones sobre NdCyT y de las habilidades de PC) que queda asociada a la función del maestro como mediador. Así pues, se emplea una evaluación formativa comunicativo-crítica y procesual (Rotger, 1990; Quinquer, 2000) que consiste en una evaluación que forma parte del proceso educativo, de carácter global, donde se generan continuos espacios para la aportación de feed-back (tanto por parte del maestro, como por los propios alumnos-compañeros) que permitan a cada estudiante la reconstrucción de su conocimiento. Este proceso otorga mucha importancia a la construcción personal del discente, a las mediaciones entre todas las personas implicadas en el proceso de enseñanza-aprendizaje y a la autonomía y el razonamiento del alumnado (Ortega-Quevedo, Santamaría-Cárdaba y Gil, 2017). La implementación de los instrumentos de evaluación prefijados para esta investigación son complementos de esta evaluación que miden de una forma precisa los factores de estudio.

Asimismo, el cómputo de estrategias didácticas y las actividades que conforman la SEA toman como base la estructura didáctica denominada ciclo de aprendizaje 7E que propone siete fases para la enseñanza de las ciencias naturales (extraer, enganchar, Explorar, explicar, elaborar, extender y evaluar) (Eisenkraft, 2003).

\section{Planificación y organización de contenidos}

\section{Instrumentos de evaluación}

Con el fin de identificar las concepciones sobre $\mathrm{NdCyT}$ que tienen los participantes, se han adaptado algunos ítems del cuestionario de opiniones sobre ciencia, tecnología y sociedad (COCTS) (Vázquez, Manassero y Acevedo, 2006). Dicho cuestionario es conocido como la versión española del Views on Science-Technology-Society (VOSTS) (Aikenhead y Ryan, 1989) y del Teacher's Belief about Science-Technology-Society (TBASTS) (Rubba y Harkness, 1993). En concreto, se han adaptado cuatro ítems del COCTS relacionados con los cuatro temas de NdCyT que se trabajan (tabla 3).

La validación de esta adaptación se ha realizado en tres fases. La primera ha consistido en la evaluación del cuestionario por un comité de tres expertos en la temática, la segunda ha radicado 
Tabla 2. Planificación y organización de contenidos en la SEA.

\begin{tabular}{|c|c|c|c|c|c|}
\hline & Sesión & & 1 & 2 & 3 \\
\hline \multirow{9}{*}{ Actividad } & \multirow{3}{*}{ Inicial } & Descripción & $\begin{array}{l}\text { Rutina de pensamiento: generar, } \\
\text { clasificar, conectar y elaborar }\end{array}$ & \multicolumn{2}{|l|}{$\begin{array}{l}\text { Rutina de pensamiento: } \\
\text { veo, pienso, me } \\
\text { pregunto }\end{array}$} \\
\hline & & $\begin{array}{l}\text { Contenidos } \\
\text { curriculares }\end{array}$ & $\begin{array}{l}\text { Concepto de energía, } \\
\text { propiedades de la energía y } \\
\text { manifestaciones de la energía }\end{array}$ & \multicolumn{2}{|l|}{$\begin{array}{l}\text { Impactos ambientales } \\
\text { relacionados con la } \\
\text { energía }\end{array}$} \\
\hline & & $\begin{array}{l}\text { Contenidos } \\
\text { transversales } \\
\text { del estudio }\end{array}$ & $\begin{array}{l}\text { Influencia de la ciencia y la } \\
\text { tecnología en la sociedad } \\
\text { (ICTS), razonamiento verbal, } \\
\text { argumentación }\end{array}$ & \multicolumn{2}{|l|}{$\begin{array}{l}\text { ICTS razonamiento } \\
\text { verbal, argumentación y } \\
\text { probabilidad }\end{array}$} \\
\hline & \multirow{3}{*}{ Desarrollo } & Descripción & \multicolumn{3}{|c|}{$\begin{array}{l}\text { Se genera un diálogo con el alumnado (escuchan preguntan) donde ser presentan los } \\
\text { contenidos de forma contextualizada y se insta al alumnado a opinar sobre los mismos. }\end{array}$} \\
\hline & & $\begin{array}{l}\text { Contenidos } \\
\text { curriculares }\end{array}$ & $\begin{array}{l}\text { Concepto de energía, } \\
\text { propiedades de la energía y } \\
\text { manifestaciones de la energía }\end{array}$ & $\begin{array}{l}\text { Impactos ambientales } \\
\text { relacionados con la } \\
\text { energía, y fuentes de } \\
\text { energías renovables y } \\
\text { no renovables }\end{array}$ & $\begin{array}{l}\text { Fracturación } \\
\text { hidráulica y sus } \\
\text { consecuencias }\end{array}$ \\
\hline & & $\begin{array}{l}\text { Contenidos } \\
\text { transversales } \\
\text { del estudio }\end{array}$ & $\begin{array}{l}\text { ICTS, razonamiento verbal, } \\
\text { argumentación }\end{array}$ & $\begin{array}{l}\text { ICTS, razonamiento } \\
\text { verbal, argumentación y } \\
\text { probabilidad }\end{array}$ & $\begin{array}{l}\text { ICTS, construcción } \\
\text { social de la } \\
\text { tecnología (CST), } \\
\text { razonamiento verbal, } \\
\text { argumentación y } \\
\text { probabilidad }\end{array}$ \\
\hline & \multirow{3}{*}{ Consolidación } & Descripción & \multicolumn{3}{|c|}{$\begin{array}{l}\text { Se realizan ejercicios por parejas que contiene preguntas que inducen a la } \\
\text { reflexión. Seguido se ponen en común dichas respuestas generando debates, donde } \\
\text { el proceso argumental promueve el contraste de hipótesis y su reformulación, junto } \\
\text { con la reconstrucción de los conocimientos de los estudiantes }\end{array}$} \\
\hline & & $\begin{array}{l}\text { Contenidos } \\
\text { curriculares }\end{array}$ & $\begin{array}{l}\text { Concepto de energía, } \\
\text { propiedades de la energía y } \\
\text { manifestaciones de la energía }\end{array}$ & $\begin{array}{l}\text { Impactos ambientales } \\
\text { relacionados con la } \\
\text { energía, y fuentes de } \\
\text { energía renovables y no } \\
\text { renovables }\end{array}$ & $\begin{array}{l}\text { Fracturación } \\
\text { hidráulica y sus } \\
\text { consecuencias }\end{array}$ \\
\hline & & $\begin{array}{l}\text { Contenidos } \\
\text { transversales } \\
\text { del estudio }\end{array}$ & $\begin{array}{l}\text { ICTS, razonamiento verbal, } \\
\text { argumentación }\end{array}$ & $\begin{array}{l}\text { ICTS, razonamiento } \\
\text { verbal, argumentación y } \\
\text { probabilidad }\end{array}$ & $\begin{array}{l}\text { ICTS, razonamiento } \\
\text { verbal, } \\
\text { argumentación y } \\
\text { probabilidad }\end{array}$ \\
\hline
\end{tabular}

Fuente: Elaboración propia de los autores.

Tabla 3. Ítems de la adaptación del COCTS en relación con la temática de NdCyT.

\begin{tabular}{lc}
\hline Temática de NdCyT & Ítem de identificación \\
Influencia de la ciencia y la tecnología en la sociedad & 40211 \\
Influencia de la ciencia y la tecnología en la sociedad & 40311 \\
Construcción social de la tecnología & 80131 \\
Construcción social de la tecnología & 80211 \\
\hline
\end{tabular}

Fuente: Elaboración propia de los autores. 
en la evaluación del lenguaje expuesto en el instrumento por parte de tres maestros de educación primaria y la tercera se centra en la comprobación estadística de la fiabilidad mediante las pruebas de: estabilidad temporal (test-retest), consiguiendo índices de fiabilidad en los enunciados entre 0.605 y 0.95 ; y alfa de Cronbach con resultado 0.65 .

El método de respuesta aplicado a esta adaptación mantiene el del cuestionario original. Dicho método se ajusta a un modelo de respuesta múltiple, en el cual los participantes deben valorar su grado de acuerdo o desacuerdo, expresado en una escala del 1 al 9, con cada enunciado contenido en los ítems (Manassero, Vázquez y Acevedo, 2003). En lo relativo a la métrica, es necesario esclarecer que para interpretar las respuestas directas como medidas actitudinales se ha tomado como referencia una baremación que clasifica los distintos enunciados alternativos en tres categorías: adecuada (cuando la frase expresa una opinión adecuada sobre la actitud), plausible (cuando la frase no es totalmente adecuada, pero expresa algunos aspectos apropiados) e ingenua (cuando la frase presenta un punto de vista que no es ni adecuado ni plausible) (Vázquez, Acevedo y Manassero, 2000). Asimismo, para determinar a qué categoría pertenece cada uno de los enunciados una veintena de expertos en la temática realizó el cuestionario. De este modo, se llega a establecer que las respuestas inadecuadas son aquellas que están por debajo del valor 4, las respuestas plausibles son las que se encuentran entre el 4 y el 6 , y las adecuadas son aquellas que superaban el 6 (Vázquez et al., 2000).

Del mismo modo, con el objetivo de evaluar las habilidades de PC, se han adaptado algunas situaciones de una traducción del Halpern Critical Thinking Assessmentusing Everyday Situations (HCTAES) (Halpern, 1998) facilitada por el proyecto CYTPENCRI. En concreto, se han adaptado tres situaciones del cuestionario relacionadas con los factores de interés (tabla 4).
Tabla 4. Situaciones de la adaptación de la versión española de la prueba Halpern en relación con los factores de PC.

\begin{tabular}{lc}
\hline Habilidades de PC & $\begin{array}{c}\text { Situación del cuestionario } \\
\text { español original }\end{array}$ \\
Razonamiento verbal & Situación 10 \\
Argumentación & Situación 12 \\
$\begin{array}{l}\text { Probabilidad/comprobación } \\
\text { de hipótesis }\end{array}$ & Situación ejemplo \\
\hline
\end{tabular}

Fuente: Elaboración propia de los autores.

La validación de esta adaptación se ha realizado siguiendo las fases aplicadas con el COCTS: validación de contenido por expertos, del lenguaje por maestros y estadística. En este caso, la comprobación estadística de la fiabilidad del cuestionario muestra un índice 0.69 y 0.95 en las pruebas de test-restest y 0.551 en el alfa de Cronbach.

En lo relativo a la métrica de este instrumento, es necesario esclarecer los criterios de corrección. Estos consisten en una forma de otorgar una determinada puntuación por pregunta diferenciando las partes abiertas de las partes cerradas de cada situación. Concretamente, en la parte abierta si los participantes dan una respuesta correcta se les concede dos puntos, si la respuesta es aproximada pero poco explícita se concede un punto, y si la respuesta es incorrecta no puntúa. En la parte cerrada se pide a los participantes que elijan una serie de respuestas, de modo que por cada una de las respuestas correctas seleccionadas se les concede un punto (Nieto, Saiz y Orgaz, 2009).

\section{Resultados y Discusión}

En este apartado se presenta el análisis descriptivo e inferencial de los datos recogidos en los cuestionarios empleados en la investigación.

\section{Concepciones sobre NdCyT}

Con el fin de comprobar la evolución en las concepciones de los estudiantes de los grupos experimentales sobre los temas de NdCyT seleccionados, se recogen en la figura 1 diagramas de cajas (DC) 
que muestran la distribución de las respuestas de los participantes en las fases de pre y post-test, así como la interpretación correcta de los enunciados según los expertos (ICEE) de las mismas (aceptable [Ac], plausible [P] e ingenua [I]).

Para interpretar esta imagen, así como las restantes, es preciso observar cómo la distribución inicial de las respuestas de los participantes para cada uno de los enunciados cambia en la fase post-test. Esta variación en la distribución aproxima las respuestas de los estudiantes al intervalo prefijado por los expertos como correcto.
A modo de ejemplo, se interpreta el enunciado A del ítem 40211. En lo relativo al DC del pre-test, el bigote superior del primer diagrama se extiende desde el valor 8 al 9, lo cual indica que el $25 \%$ de los participantes han señalado dichos valores; por el contrario, el bigote inferior se extiende desde el 1 al 5, permitiendo observar una menor concentración en las respuestas de menor valoración por otro $25 \%$ de los participantes. La media se sitúa en el valor 6 y divide la caja mostrando mayor concentración de respuestas entre los valores 5 y 6 que entre los valores 6 y 8 . En cuanto al DC del

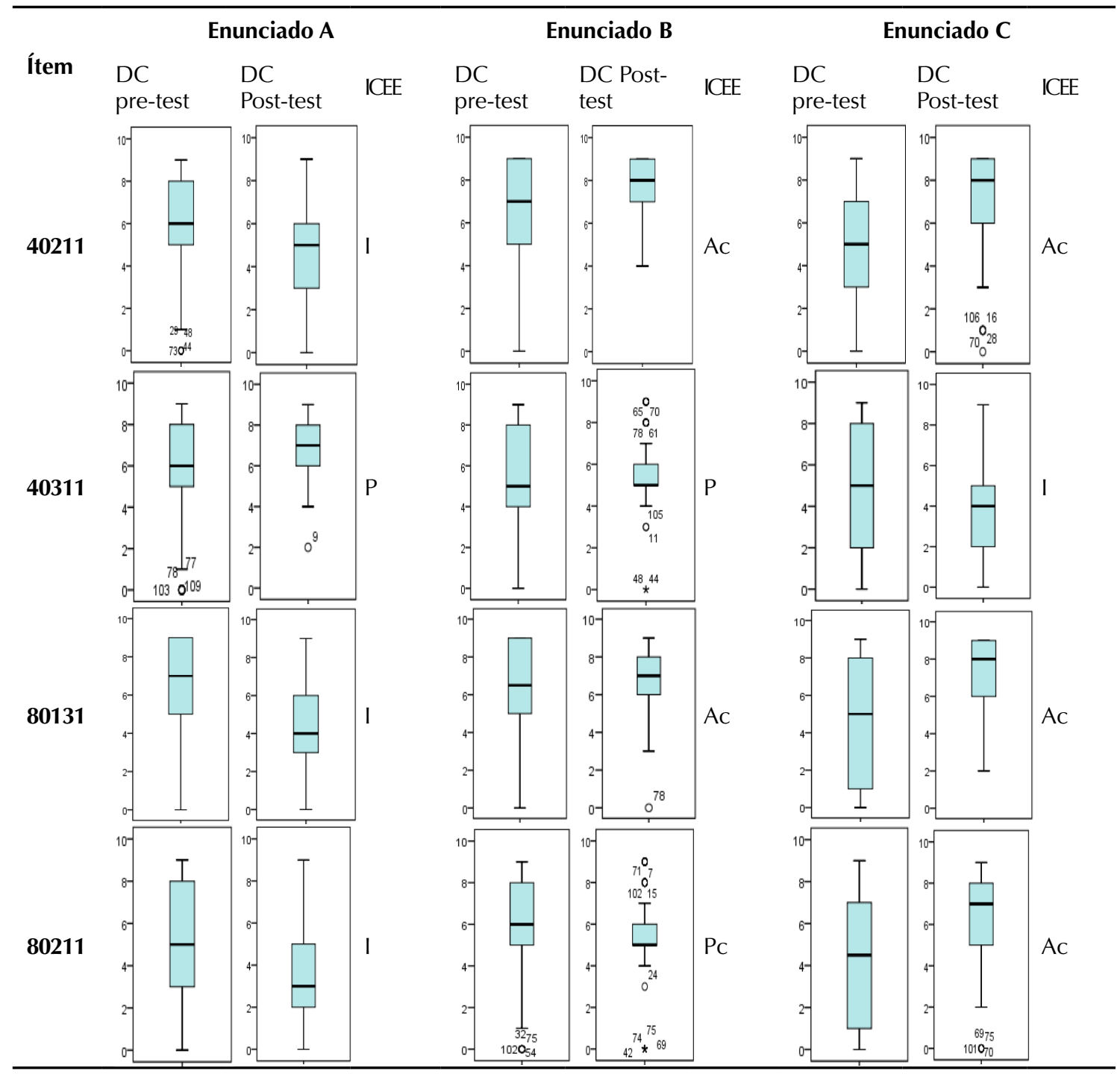

Figura 1. Resultados descriptivos de la adaptación del COCTS. Grupos experimentales. 
post-test, se observa un importante cambio en la extensión del bigote superior que indica cómo el $25 \%$ de los participantes dan su valoración entre el 8 y el 6. La extensión del bigote inferior también se ha modificado alcanzando valores entre el 0 y el 3. En este caso la media se sitúa en el 5 y divide la caja entre los valores 3 y 6 , siendo mayor la concentración de respuestas entre el 5 y el 6 .

Así pues, la distribución de las respuestas del alumnado del post-test queda más próxima al intervalo de respuestas marcado por los expertos como correctos, que en este caso sería del 0 al 4, al ser la respuesta clasificada como I (ingenua).
Por otra parte, con el fin de establecer si las diferencias entre las distribuciones de cada uno de los enunciados de los ítems son significativas según los periodos de investigación (pre y post-test) se ha realizado la prueba Anova de dos vías de Friedman asumiendo un error del $5 \%$. Dichas pruebas determinan que las diferencias entre la distribución de todos los enunciados son estadísticamente significativas a excepción del enunciado $B$ del ítem 40311. En el caso de este ítem se observa una asignación de los valores entre el 7 y el 4, que se ajusta a lo establecidos por los expertos como correcto. Sin embargo, los cambios en la distribución no son significativos estadísticamente.

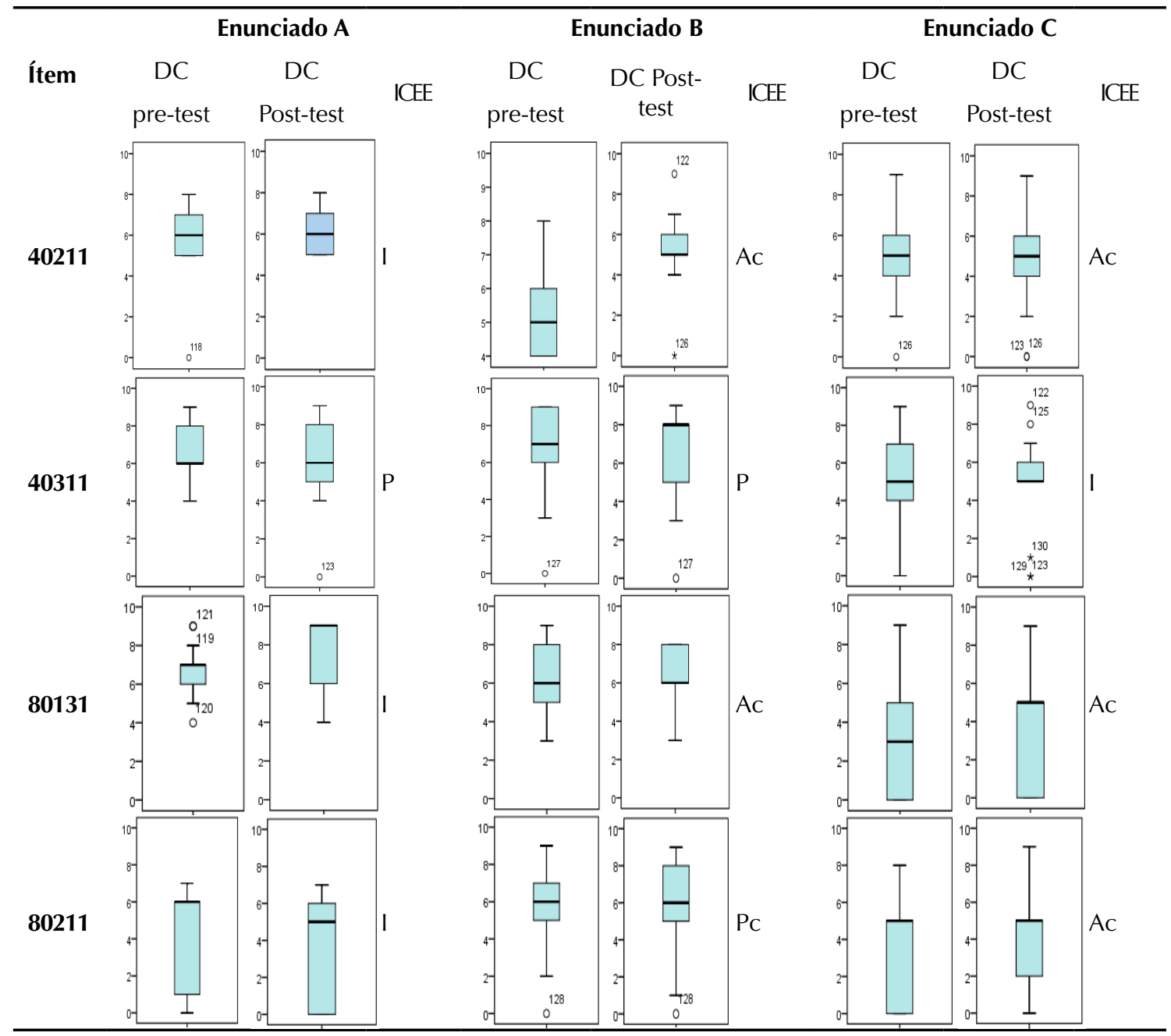

Figura 2. Resultados descriptivos de la adaptación del COCTS. Grupo control. 
Una vez analiza la evolución en la corrección de las respuestas de los participantes de los grupos experimentales sobre los ítems de NdCyT se procede a estudiar la evolución de las concepciones del grupo control con los resultados recogidos en la figura 2.

En el caso del grupo control, se observa cómo la distribución marcada en los DC no presenta cambios aparentemente destacables que muestren una reconducción de las concepciones sobre NdCyT del alumnado entre los periodos pre y post-test. La significatividad en la diferencia de distribuciones de los enunciados de cada uno de los ítems entre los periodos pre y post-test se ha comprobado de nuevo mediante la prueba Anova de dos vías de Friedman. Los resultados a dicha prueba determinan que las diferencias son significativas únicamente en el enunciado A del ítem 80131. No obstante, si se analizan los DC se puede observar cómo en la fase post-test los valores del alumnado se desvían del intervalo correcto, según los expertos (4-6).

Finalmente, se destaca la reconducción de las concepciones de NdCyT de los participantes del grupo experimental hacia lo establecido por los expertos como correcto durante la fase post-test y la ausencia de esta en lo que respeta a los participantes del grupo control. Esto prueba la contribución de la implementación de la SEA en la mejora de las concepciones.

\section{Factores de PC}

Del mismo modo que en el apartado anterior, en la figura 3 se presentan los resultados de los estudiantes de los grupos experimentales en las fases pre y post-test. Dicha tabla contiene DC de las fases de pre y post-test y la puntuación máxima (PM) que se puede obtener en cada parte del cuestionario; la interpretación de los DC en cuanto a la interpretación de la distribución se debe realizar de la misma forma que en el ejemplo presentado anteriormente.
En lo relativo al análisis inferencial se exponen las diferencias estadísticamente significativas según los resultados obtenidos en las pruebas Anova de dos vías de Friedman, aplicadas a cada una de las partes de las distintas situaciones en las fases pre y post-test. Dichas pruebas muestran la existencia de diferencias significativas en las partes cerradas de las tres situaciones. Sin embargo, en el caso de las partes abiertas solamente se encuentra una mejora notable en la tercera situación. Estos resultados determinan que hay mejoras importantes en las habilidades de razonamiento verbal y argumentación, cuando los participantes reciben una guía para realizar el proceso de PC y que los discentes han mejorado la habilidad relacionada con la probabilidad tanto de forma guiada como abierta.

Por otra parte, en la figura 4 queda reflejada la reducida evolución en la mejora de los factores de PC estudiados en los participantes relativos al grupo control. La significatividad en la diferencia de distribuciones de los enunciados de cada parte de las situaciones entre los periodos pre y post-test se ha comprobado de nuevo mediante la prueba Anova de dos vías de Friedman. Los resultados de dicha prueba determinan que las diferencias son significativas únicamente en la parte abierta de la segunda situación, donde la distribución indica una mejora en el factor de argumentación en la parte no guiada.

\section{Conclusiones}

Los participantes de los grupos experimentales en la fase de post-test han demostrado una mejora en sus concepciones sobre los factores de estudio relacionados con la NdCyT. Esta mejora es significativa en todos los enunciados de los ítems a excepción de uno. Pero, dicha mejora no se aprecia del mismo modo en la evolución de las concepciones de los estudiantes pertenecientes al grupo control. Esto permite evidenciar cómo la SEA ha contribuido a la mejora de las concepciones del alumnado de los grupos experimentales. 
Los discentes de los grupos experimentales han mejorado de forma significativa sus habilidades de PC en todas las dimensiones de estudio manteniendo una guía a la hora de aplicar las medidas (partes cerradas de las situaciones). Además, en la dimensión de probabilidad se ha alcanzado una mejora en las habilidades de estos estudiantes también sin guía (parte abierta de las situaciones). Sin embargo, los estudiantes del grupo control solo han mostrado mejoras durante la realización de la prueba abierta del factor argumentación. Esto muestra de nuevo el valor de la implementación de la SEA para el desarrollo de estas habilidades.

Como se ha señalado, la SEA diseñada ha contribuido a la mejora de las concepciones de los temas de NdCyT seleccionados para el estudio, así como al desarrollo de las dimensiones de PC trabajadas. Esta contribución prueba la validez de la SEA.

De acuerdo con lo expuesto, se concluye que el aprendizaje y desarrollo alcanzado por los participantes de los grupos experimentales, tras la puesta en práctica de la SEA de tres sesiones, hace ver el potencial de este tipo de diseños didácticos en las aulas de educación primaria. Por este motivo se considera necesario el desarrollo e implementación de este tipo de estudios en esta etapa, con el fin de profundizar en sus resultados y contrastar la potencialidad de los mismos en el desarrollo conjunto de las competencias en ciencia, tecnología y pensamiento crítico de los estudiantes.

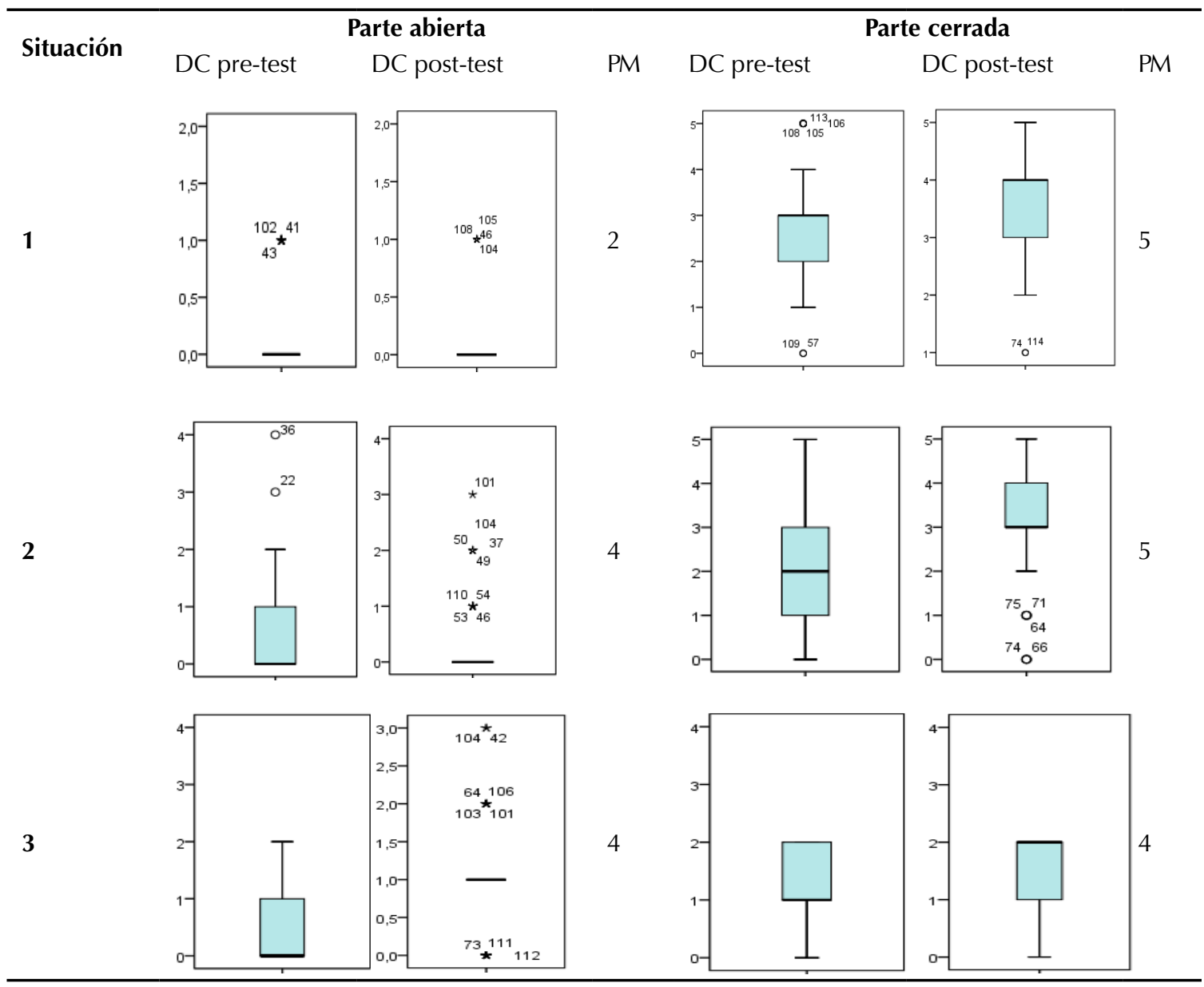

Figura 3. Resultados descriptivos de la adaptación del test de Halpern. Grupos experimentales. 


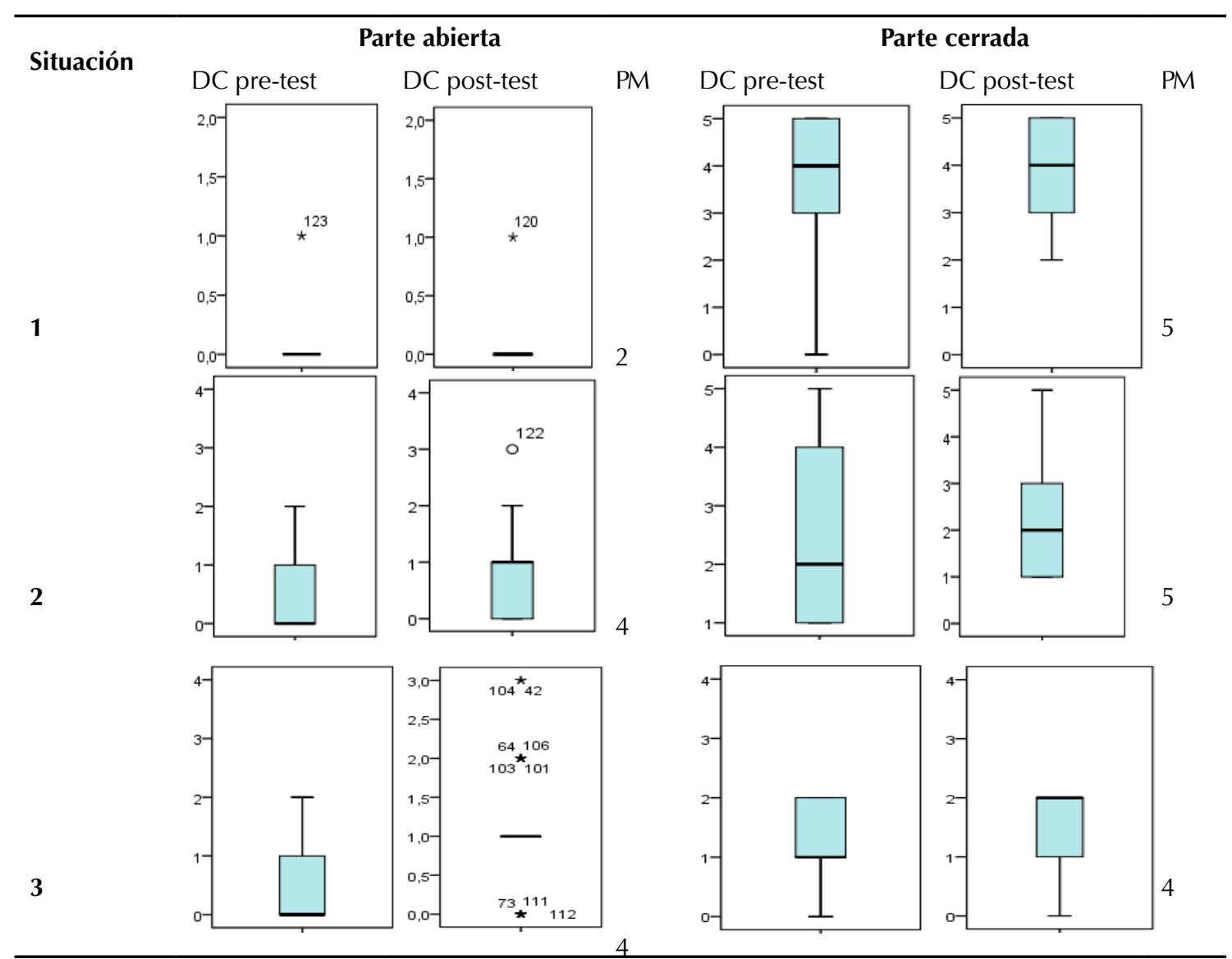

Figura 4. Resultados descriptivos de la adaptación del test de Halpern. Grupo control.

\section{Agradecimientos}

La autoría agradece al proyecto EDU201564642-R (MINECO/FEDER) con financiación del Ministerio de Economía y Competitividad de España y el Fondo Europeo de Desarrollo Regional su colaboración.

\section{Referencias}

Abd-El-Khalick, F. (2012). Examining the sources for our understandings about science: enduring conflations and critical issues in research on nature of science in science education. International Journal of Science Education, 34(3), 353-374. https://doi.org/10.1080/09500693.2 $\underline{011.629013}$
Acevedo, J. A. (2009). Enfoques explícitos versus implícitos en la enseñanza de la naturaleza de la ciencia. Revista Eureka sobre Enseñanza y Divulgación de las Ciencias, 6(3), 355-386. https://doi.org/10.25267/Rev Eureka ensen divulg_cienc.2009.v6.i3.04

Acevedo, J. A.; Aragón-Méndez, M. M.; García-Carmona, A. (2018). Comprensión de futuros profesores de ciencia sobre aspectos epistémicos de la naturaleza de la ciencia en cuatro controversias de historia de la ciencia. Revista Científica, 33(3), 344-355. https://doi. org/10.14483/23448350.13355

Acevedo, J. A.; García-Carmona, A. (2016). Algo antiguo, algo nuevo, algo prestado. Tendencias sobre la naturaleza de la ciencia en la educación científica. Revista Eureka sobre 
Enseñanza y Divulgación de las Ciencias, 13(1), 3-19. https://doi.org/10.25267/Rev Eureka ensen divulg_cienc.2016.v13.i1.02

Acevedo, J. A.; García-Carmona, A.; Aragón-Méndez, M. M. (2016) Un caso de Historia de la Ciencia para aprender Naturaleza de la Ciencia: Semmelweis y la fiebre puerperal. Revista Eureka sobre Enseñanza y Divulgación de las Ciencias, 13(2), 408-422. https://doi. org/10.25267/Rev Eureka ensen divulg cienc.2016.v13.i2.13

Aikenhead, G. S.; Ryan, A. G. (1989). The development of a multiple choice instrument for monitoring views on Science-Technology-Society topics. Final Report of SSHRCC Grant. Saskatoon, Canadá: Universidad de Saskatchewan.

Deng, F.; Chen, D. T.; Tsai, C. C.; Chai, C. S. (2011). Students' Views of the Nature of Science: A Critical Review of Research. Science Education, 95, 961-999. https://doi.org/10.1002/ $\underline{\text { sce. } 20460}$

Dochy, F.; Segers, M.; Dierick, S. (2002). Nuevas vías de aprendizaje y enseñanza y sus consecuencias: una nueva era de evaluación. Docencia universitaria, 2(2), 1-29.

Eisenkraft, A. (2003). Expanding the 5E model: A proposed 7E model emphasizes "transfer of learning" and the importance of eliciting prior understanding. The Science Teacher, 70, 56-59.

Ennis, R. H. (1996). Critical Thinking. Nueva Jersey: Prentice-Hall.

Facione, P. A. (1990). Critical thinking: A statement of expert consensus for purposes of educational assessment and instruction. Executive Summary "The Delphi Report".

Franco, A.; Almeida, L.; Morales, L. (2014). Pensamiento crítico: reflexión sobre su lugar en la Enseñanza Superior. Educación del siglo XXI, 32(2), 81-96. https://doi.org/10.6018/j/202171

Garrido, J. M.; Perales, F. J.; Galdón, M. (2007). Ciencia para educadores. Madrid: Pearson Prentice Hall.
Gold, K. (2002). Thinking: the next big idea. TES:Website. TES: Website more than a job. https://www.tes.com/news/ thinking-next-big-idea

González, F. (coord.); Álvarez, P.; Carrillo, F. J.; García-Alix, A.; Jiménez, M. P.; Romero, C.; Ruiz, L. (2015). Didáctica de las ciencias para educación primaria II. Ciencias de la vida. Madrid: Pirámide.

Halpern, D. F. (1998). Teaching critical thinking for transfer across domains. American Psychologist, 53(4), 449-455. https://doi. org/10.1037/0003-066X.53.4.449

Horizonte 2020 para la Investigación e Innovación en la Unión Europea (s. f.). Ciencia con y para la sociedad. https://eshorizonte2020.es/ mas-europa/ciencia-con-y-para-la-sociedad

Jefatura del Estado de España (9 de diciembre de 2013). Ley Orgánica 8, para la mejora de la calidad educativa España. Madrid.

López, G. (2012). Pensamiento crítico en el aula. Docencia e Investigación, 22, 41-60.

Manassero, M. A.; Vázquez, A. (septiembre de 2017). ¿Hay contenidos de naturaleza de la ciencia y la tecnología y pensamiento crítico en los currículos (españoles) actuales? $X$ Congreso Internacional sobre Investigación en Didáctica de las Ciencias. Sevilla, España.

Manassero, M. A.; Vázquez, A.; Acevedo, J. A. (2003). Cuestionario de opiniones sobre ciencia, tecnologia i societat (COCTS). Princeton, NJ: Educational Testing Service. http://www. ets.org/testcoll/

Martín, C.; Prieto, T.; Jiménez, M. A. (2015). Tendencias del profesorado de ciencias en formación inicial sobre las estrategias metodológicas en la enseñanza de las ciencias. Estudio de un caso en Málaga. Enseñanza de las Ciencias, 33(1), 167-184. http://dx.doi.org/10.5565/rev/ ensciencias. 1500

Nieto, A. M.; Saiz, C.; Orgaz, B. (2009). Análisis de las propiedades psicométricas de la versión española del HCTAES-Test de Halpern para la 
evaluación del pensamiento crítico mediante situaciones cotidianas. Revista Electrónica de Metodología Aplicada, 14(1), 1-15.

Norris, S. P. (1985). The Choice of Standard Conditions in Defining Critical Thinking Competence. Educational Theory, 35, 97-107. https:// doi.org/10.1111/j.1741-5446.1985.00097.x

Ortega-Quevedo, V.; Santamaría-Cárdaba, N.; Gil, C. (2017). La evaluación formativa en una secuencia de enseñanza aprendizaje de ciencias experimentales. Infancia, Educación y Aprendizaje, 3(2), 203-209. http://dx.doi.org/10.22370/ ieya.2017.3.2.724

Paul, R. (2005). The state of critical thinking today. New Directions for Community Colleges, Summer 2005, 27-38. https://doi.org/10.1002/ $\underline{\text { CC. } 193}$

Paul, R.; Elder, L. (2002). Critical thinking: Tools for taking charge of your professional and personal life. Upper Saddle River: Pearson Education.

Pedrinaci, E. (coord.), Caamaño, A., Cañal, P. y de Pro, A. (2012). 11 ideas clave. El desarrollo de la competencia científica. Barcelona: Graó

Quinquer, D. (2000). Modelos y enfoques sobre evaluación: el modelo comunicativo. En A. Parcerisa (dir.), Evaluación como ayuda al aprendizaje (pp.13-20). Barcelona: Laboratorio Educativo, Graó.

Real Decreto 126/2014, de 28 de febrero, por el que se establece el currículo básico de la Educación Primaria, pp. 19349-19420.

Rotger, B. (1990). Evaluación formativa. Madrid: Cincel.

Rubba, P. A.; Haskness, W. L. (1993). Examination of Pre-service and In-Service Secondary Science teachers' beliefs about Science-Technology-Society interactions. Science Education, 77(4), 407-431. https://doi.org/10.1002/ sce.3730770405

Saiz, C. (2002). Enseñar o aprender a pensar. Escritos de Psicología, 6, 53-72.

da Silva, L.; y Rodríguez, A. H. (2011). Critical thinking: Its relevance for education in a shifting society. Revista de Psicología, 29(1), 195-195.
Solbes, J.; Montserrat, R.; Furió, C. (2007). El desinterés del alumnado hacia el aprendizaje de la ciencia: implicaciones en su enseñanza. Didáctica de las ciencias experimentales y sociales, 21, 91-117.

Valenzuela, J.; Nieto, A. M. (2008). Motivación y pensamiento crítico: aportes para el estudio de esta relación. Revista Electrónica de Motivación y Emoción, XI(28), 1-8. http://reme.uji.es/ articulos/numero28/article3/article3.pdf

Vázquez, A.; Acevedo, J. A.; Manassero, M. A. (2000). Progresos en la evaluación de actitudes relacionadas con la ciencia mediante el Cuestionario de Opiniones CTS. En I. P. Martins (coord.), O Movimento CTS na Península Ibérica. Seminário Ibérico sobre Ciência-Tecnologia-Sociedade no ensino-aprendizagem das ciencias experimentais (pp. 219-230). Aveiro: Universidade de Aveiro.

Vázquez, A.; Manassero, M. A. (2012). La selección de contenidos para enseñar naturaleza de la ciencia y tecnología (parte 1): Una revisión de las aportaciones de la investigación didáctica. Revista Eureka sobre Enseñanza y Divulgación de las Ciencias, 9(1), 2-31. https:// doi.org/10.25267/Rev Eureka ensen divulg cienc.2012.v9.i1.02

Vázquez-Alonso, A.; Manassero-Mas, M. A. (2018). El conocimiento epistémico en la evaluación de la competencia científica en PISA 2015. Revista de Educación, 380, 103-128. https://doi. org/10.4438/1988-592X-RE-2017-380-373

Vázquez, A.; Manassero, M. A.; Acevedo, J. A. (2006). An analysis of complex multiple choice science-technology-society items: Methodological development and preliminary results. Science Education, 90(4) 681-706. https://doi. org/10.1002/sce.20134

Vázquez, A.; Manassero, M. A.; Acevedo, J. A.; y Acevedo, P. (2007). Consensos sobre la naturaleza de la Ciencia: la comunidad tecnocientífica. Revista Electrónica de Enseñanza de las Ciencias, 6(2), 331-363. 
Vázquez, A.; Manassero, M. A.; Talavera, M. (2010). Actitudes y creencias sobre naturaleza de la ciencia y la tecnología en una muestra representativa de jóvenes estudiantes. Revista
Electrónica de Enseñanza de las Ciencias, 9(2), 333-352.

Wells, G. (2001). Indagación dialógica. Barcelona: Paidós. 